\title{
Polimorfismo de tamaño entre cromosomas homólogos, un caso extremo en Alstroemeria pulchra Sims. (Alstroemeriaceae), especie endémica de Chile
}

\section{Size polymorphism between homologous chromosomes, an extreme case in Alstroemeria pulchra Sims. (Alstroemeriaceae), endemic species of Chile}

\author{
Carlos Baeza ${ }^{1 *}$, Oscar Toro ${ }^{1}$, Eduardo Ruiz ${ }^{1}$, Victor Finot ${ }^{2}$, Pedro Carrasco ${ }^{1}$ \& Nicolás \\ VILLALOBOS $^{2}$
}

${ }^{1}$ Facultad de Ciencias Naturales y Oceanográficas, Departamento de Botánica, Casilla 160-C, Universidad de Concepción, Concepción, Chile.

${ }^{2}$ Departamento de Producción Animal, Facultad de Agronomía, Universidad de Concepción, Chillán, Chile.

*cbaeza@udec.cl

\begin{abstract}
Alstroemeria pulchra is a Chilean species where 3 subspecies are recognized: subsp. lavandulacea, maxima and pulchra. The chromosomes of populations of the subspecies lavandulacea and pulchra were studied and a marked size polymorphism was observed among the homologous of the first pair of chromosomes. This size polymorphism among homologous is one of the rarest reported thus far in the Angiosperm literature.
\end{abstract}

El género Alstroemeria incluye aproximadamente 80 especies endémicas de Sudamérica, con dos centros de diversidad (Chile y Brasil). En Chile, este género representa uno de los grupos más diversos de monocotiledóneas, con más de 50 taxones reconocidos o aceptados (36 especies, 11 subespecies y 10 variedades), de los cuales ca. del $82 \%$ son endémicos de la zona mediterránea de Chile central, uno de los hotspots de diversidad del mundo (Finot et al. 2018). Muñoz \& Moreira (2003) reconocen numerosos "complejos" dentro del género, siendo uno de estos Alstroemeria pulchra Sims. En este complejo se reconocen tres subespecies: subsp. pulchra (Fig. 1A), subsp. lavandulacea Ehr. Bayer (Fig. 1B) y subsp. maxima Phil. Crecen desde el cerro Pan de Azúcar al sur de Coquimbo (2959'S) hasta la cordillera de Nahuelbuta, en la Región de la Araucanía (37²4'S).

Los estudios citogenéticos en Alstroemeria son útiles para la delimitación taxonómica, ya que cada especie, subespecie o variedad se caracteriza por poseer un cariotipo único (Baeza et al. 2007). Estos estudios han contribuido a la delimitación de taxones a escalas inter e intraespecíficas, así como a la comprensión de los procesos cromosómicos que determinan la divergencia entre ellos (Baeza et al. 2007). En muchas especies de Alstroemeria se ha encontrado polimorfismo entre homólogos, tanto en el contenido de ADN nuclear como en la cantidad de bandas C de heterocromatina (Buitendijk \& Ramanna1996, Buitendijk et al.1997). El mismo polimorfismo se ha encontrado en la ubicación de genes ribosomales entre pares de homólogos mediante hibridización in situ por fluorescencia (Baeza et al. 2007). Sin embargo, la presencia de reportes de polimorfismo entre homólogos relacionados con el tamaño de ellos es rara tanto en Alstroemeria como en Angiospermas en general. Por lo tanto, el objetivo de esta comunicación es reportar el polimorfismo conservado en el tamaño de un par de cromosomas homólogos en dos de las subespecies de $A$. pulchra, endémica de Chile.

Se recolectaron dos poblaciones de Alstroemeria pulchra: una de la subsp. lavandulacea y otra de la subsp. pulchra. Los ejemplares fueron cultivados en invernadero de la Facultad de Ciencias Naturales y Oceanográficas $\mathrm{y}$ vouchers fueron depositados en el herbario de la Universidad de Concepción (CONC): Alstroemeria pulchra Sims. subsp. pulchra. Región de Valparaíso. Prov. Marga Marga. Quebrada Estero Margarita, $122 \mathrm{~m}$, 3304'43"S-71²5'38"O, 7-X-2017, P. Carrasco 113. Alstroemeria pulchra Sims. subsp. lavandulacea. Región del Maule. Prov. Talca. Río Claro, $300 \mathrm{~m}$ al norte del puente Río Claro, desde el sur, km 216, 190 m, 35¹0'53"s; 71²3'19'O, 9-XI-2013, C. Baeza 4393 a.

La metodología de trabajo consistió en disectar puntas de raíces de 1-2 $\mathrm{cm}$ de longitud a partir del material cultivado en invernadero, las cuales fueron cortadas y pretratadas con una solución de 8-hidroxiquinolina $(2 \mathrm{mM})$ por $24 \mathrm{~h}$ a $4{ }^{\circ} \mathrm{C}$. Luego, se fijaron en una mezcla de etanol/ 
ácido acético (3:1) durante $24 \mathrm{~h}$. Se realizaron aplastados de puntas de raíces efectuando previamente una hidrólisis ácida con $\mathrm{HCl} 0,5 \mathrm{~N}$ durante $17 \mathrm{~min}$ a $42^{\circ} \mathrm{C}$. Luego se lavó el material y se tiñieron las puntas de raíz con orceína al $1 \%$. Los cromosomas se fotografiaron en un microscopio Zeiss Axioskop con cámara de video incorporada. Las placas metafásicas fueron medidas con la ayuda del programa computacional "MicroMeasure 3.3" (Reeves 2001). A partir de 20 placas metafásicas en $A$. pulchra subsp. lavandulacea y 15 placas en $A$. pulchra subsp. pulchra (5 individuos como mínimo), se midieron los cromosomas del par de homólogos número 1.
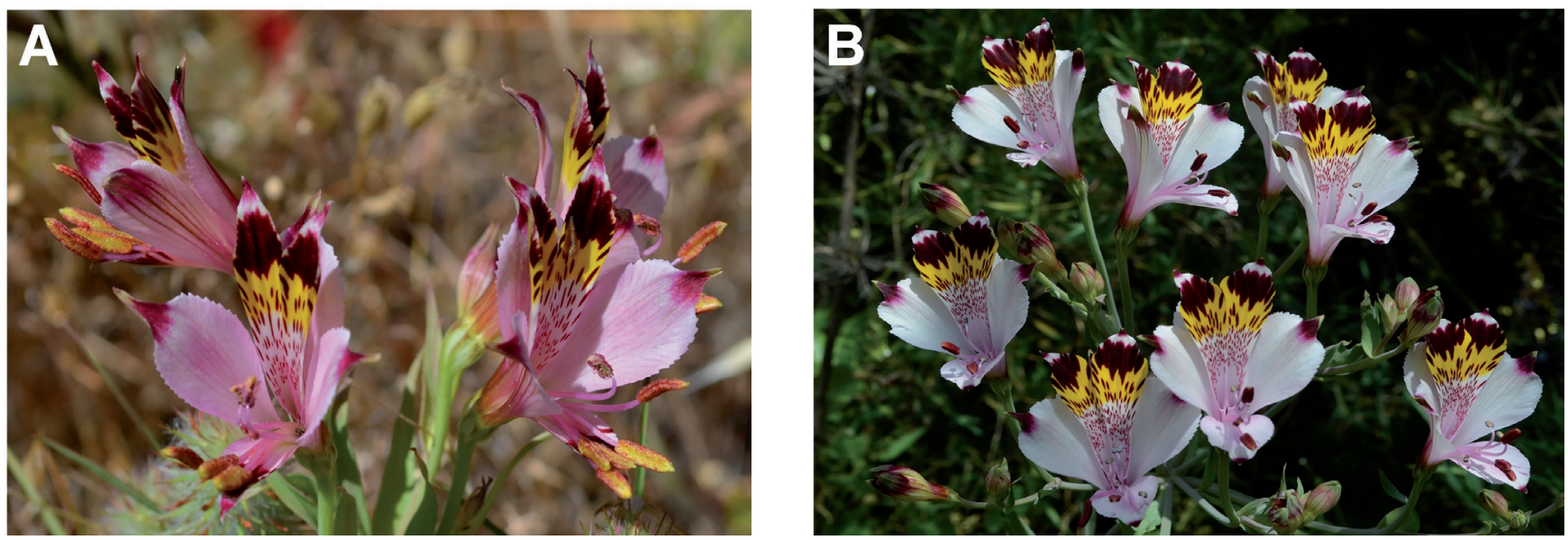

Figura 1. Fotografías de Alstroemeria pulchra. A. A. pulchra subsp. lavandulacea. B. A. pulchra subsp. pulchra. / Photographies of Alstroemeria pulchra. A. A. pulchra subsp. lavandulacea. B. A. pulchra subsp. pulchra.

Ambas poblaciones presentaron $2 \mathrm{n}=16$ cromosomas, con una fórmula cariotípica muy similar. Los resultados encontrados en ambas poblaciones evidencian un enorme polimorfismo en el tamaño de los homólogos del par de cromosomas número 1, tanto para A. pulchra subsp. lavandulacea (Fig. 2A recuadro) como para A. pulchra subsp. pulchra (Fig. 2B recuadro). La Figura 3 representa un gráfico de caja que muestra las diferencias en el tamaño de los homólogos del par cromosómico número 1 de las dos subespecies estudiadas.

El polimorfismo de tamaño entre homólogos ha sido pobremente documentado en Angiospermas. Esta situación se ha encontrado en especies de Brachycome, Triticum, Tulpia, Secale, Allium (Houben et al. 2000), Scilla (Greilhuber \& Speta 1976), Placea amoena (Baeza \& Schrader 2004) y Chaetanthera pentacaenoides (Baeza \& Torres-Díaz 2006) y también ha sido muy poco observado en Alstroemeria. Solo Buitendijk et al. (1998) encuentran este tipo de polimorfismo en poblaciones de Alstroemeria aurea Graham (en el par cromosómico número 8) y en poblaciones de A. ligtu L. (par cromosómico número 6). Baeza et al. (2018) encuentran en A. magnifica Herbert var. tofoensis M. Muñoz polimorfismo en la longitud de los brazos entre los cromosomas homólogos del par 5, que también se expresa en términos de mayores niveles de magnitud en la variación de la desviación estándar relacionada con los valores de la longitud total de los cromosomas. El reporte actual es, sin ninguna duda, el polimorfismo de tamaño entre homólogos más notable encontrado hasta ahora en Alstroemeria. En ambas subespecies la diferencia en magnitud de tamaño entre los homólogos del par cromosómico número 1 es muy evidente (Fig. 2, recuadros, Fig. 3). Esta diferencia también es demostrada con diferencias estadísticas significativas en comparaciones de largo de los cromosomas entre poblaciones $\left(\mathrm{K}-\mathrm{W} X^{2}=52.6063, \mathrm{df}=3, \mathrm{p}<0.001\right)$ y entre homólogos. La conclusión es que los valores analizados desde cromosomas designados como similares (largo vs largo y corto vs corto) son estadísticamente similares, mientras que los homólogos (largo vs corto y largo vs corto) son diferentes. Ambas observaciones fueron calculadas usando un test de KruskalWallis (Kruskal \& Wallis 1952) y un test post-hoc de Dunn (Dunn 1964), empleando el paquete estadístico dunn.test (Dinno 2017) en R v. 3.3.3 (R Core Team 2017).

En todas las especies investigadas de Alstroemeria hasta el momento el polimorfismo de tamaño se ha observado probablemente en la condición heterocigótica; no habría indicación de polimorfismo en la condición homocigota. La naturaleza de esta diferencia de compactación de la cromatina reside presumiblemente en el origen de regiones heterocromáticas, como se documenta en Brachycome (Houben et al. 2000), la que puede desempeñar un papel en la variación y el rearreglo del genoma en plantas (Jones \& Rees 1982, Navas-Castillo et al. 1987). Esta condición heterocigota representaría una ventaja selectiva para 


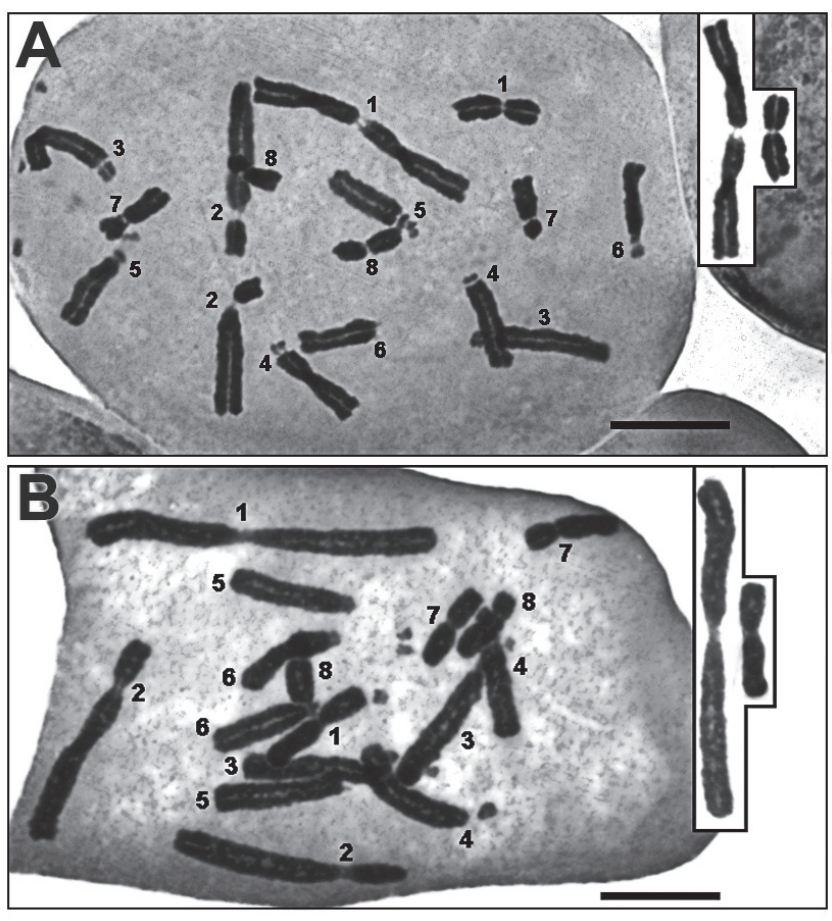

Figura 2. Placas metafásicas de Alstroemeria pulchra. A. pulchra subsp. lavandulacea. B. A. pulchra subsp. pulchra. Escala $=10$ $\mu \mathrm{m}$. / Metaphase plates of Alstroemeria pulchra. A. A. pulchra subsp. lavandulacea. B. A. pulchra subsp. pulchra. Scale $=10 \mu \mathrm{m}$.

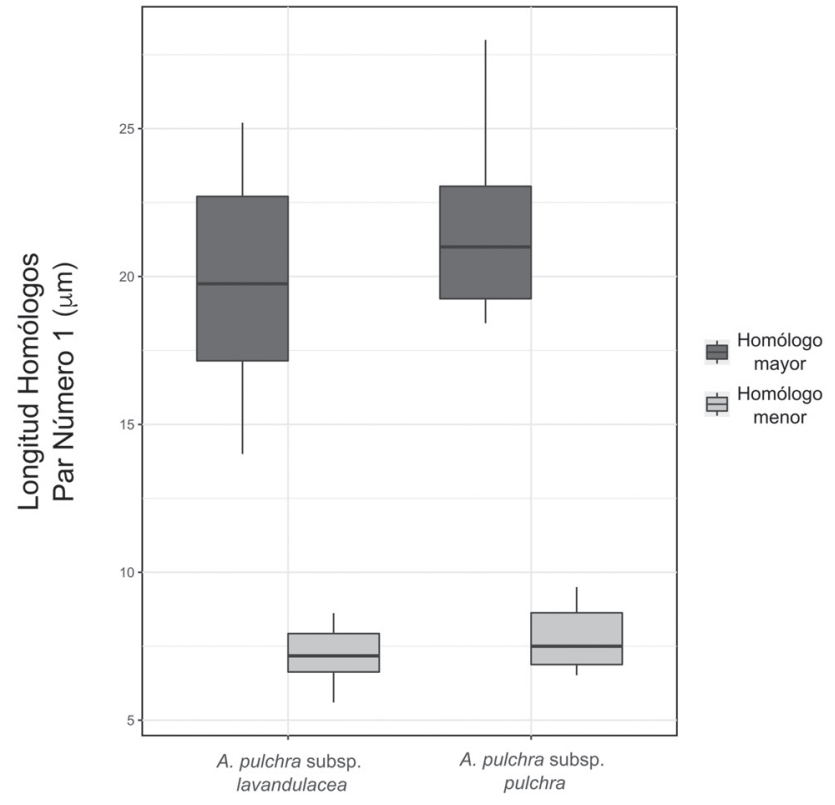

Figura 3. Gráfico de cajón del primer par de homólogos de Alstroemeria pulchra subsp. lavandulacea y A. pulchra subsp. pulchra. / Barplots of the first homologues chromosome pairs of Alstroemeria pulchra subsp. lavandulacea and A. pulchra subsp. pulchra las especies de Alstroemeria, ya que reordenamientos cromosómicos rápidos, como las translocaciones desbalanceadas recíprocas Robertsonianas (Chacon et al. 2012), podrían dar lugar a una mayor capacidad de adaptación en especies sujetas a estrés ambiental (Levin 2002). Estos procesos estarían favoreciendo un continuo proceso de reestructuración y evolución cromosómica en los genomas de Alstroemeria, los que seguirían sucediendo de forma ininterrumpida en el presente (Sanso 2002). Sin embargo, este patrón no es suficiente para explicar la persistencia de polimorfismo homocigótico en los taxones estudiados de $A$. pulchra, el que de ser originado por rearreglos debería presentar niveles distintivos de asimetría entre cromosomas homólogos (Levin 2002). Una posible explicación alternativa seria un origen por especiación híbrida, donde especies parentales hayan contribuido con cromosomas heterocigotos en su condición de largo total. Tal hipótesis contradeciría la falta de evidencia de hibridación viable en poblaciones naturales de Alstroemeria, a pesar de la reconocida facilidad de obtener híbridos cultivados vía técnicas de asistencia in vitro (Kristiansen 1995). Por lo mismo, se sugiere que mayores estudios deben realizarse sobre aspectos reproductivos de A. pulchra, además de obtener más datos desde otras poblaciones e individuos para corroborar la estabilidad del polimorfismo descrito.

\section{AGRADECIMIENTOS}

Trabajo financiado por proyecto VRID N $\mathrm{N}^{\mathrm{o}} 318 / 17$, Código 217.111.063-1.0. También se agradece al Departamento de Botánica de la Universidad de Concepción y al Proyecto Nueva Flora de Chile por todas las facilidades otorgadas para realizar este trabajo.

\section{REFERENCIAS}

Baeza, C., Schrader, O. 2004. Karyotype analysis of Placea amoena Phil. (Amaryllidaceae) by double fluorescence in situ hybridization. Caryologia 57: 209-214.

Baeza, C., Schrader, O., Budahn, H. 2007. Characterization of geographically isolated accessions in five Alstroemeria L. species (Chile) using FISH of tandemly repeated DNA sequences and RAPD analysis. Plant Systematics and Evolution 269: 1-14.

Baeza, C., Torres-DíAz, C. 2006. El cariotipo de Chaetanthera pentacaenoides (Phil.) Hauman (Asteraceae). Gayana Botánica 63: 180-182.

Baeza, C., Finot, V., Ruiz, E., Carrasco, P., Novoa, P., Rosas, M., Toro-NúÑEZ, O. 2018. Cytotaxonomic study of the Chilean endemic complex Alstroemeria magnifica Herb. (Alstroemeriaceae). Genetics and Molecular Biology 41: 434-441.

BuitendiJK, J., Ramanna, M. 1996. Giemsa C-banded karyotypes of eigth species of Alstroemeria L. and some of their 
hybrids. Annals of Botany 78: 449-457.

BuitendiJ,, J., Boon, E., Ramanna, M. 1997. Nuclear DNA content in twelve species of Alstroemeria L. and some of their hybrids. Annals of Botany 79: 343-353.

Buitendisk, J., Peters, A., Quene, R., Ramanna, M. 1998. Genome size variation and $\mathrm{C}$-band variation polymorphism in Alstroemeria aurea, A. ligtu, and A. magnifica (Alstroemeriaceae). Plant Systematics and Evolution 212: 87-106.

Chacón J., Sousa, A. Baeza, C., Renner, S. 2012. Ribosomal DNA distribution and a genus-wide phylogeny reveal patterns of chromosomal evolution in Alstroemeria. American Journal of Botany 99: 1501-1512.

Dinno, A. 2017. dunn.test: Dunn's Test of Multiple Comparisons Using Rank Sums. R package version 1.3.4. https:// CRAN.R-project.org/package $=$ dunn.test.

DunN, O. 1964. Multiple comparisons using rank sums. Technometrics 6: 241-252.

Finot, V., Baeza, C., Ruiz, E., Toro, O., Carrasco, P. 2018. Towards an Integrative Taxonomy of the genus Alstroemeria (Alstroemeriaceae) in Chile: A Comprehensive Review. In Bulent, S. (ed.). Studies in Biodiversity. Chapter 12: 229265. InterchOpen. (https://www.intechopen.com/books/ selected-studies-in-biodiversity).

Greilhuber, J., Speta, F. 1976. C-banded karyotypes in the Scilla hohenackeri group S. persica, and Puschkinia (Liliaceae). Plant Systematics and Evolution 126: 149-188.

Houben, A., Wanner, G., Hanson, L., Verlin, D., Leach, C., Timmes, J. 2000. Cloning and characterization of polymorphic heterochromatic segments of Brachycome dichromosomatica. Chromosoma 109: 206-213.

Jones, R., Rees, H. 1982. B-chromosomes. Academic Press, London. 266 pp.

KRISTIANSEN, K. 1995. Interspecific hybridization of Alstroemeria. Acta Horticulturae 420: 85-88.

Kruskal, W., Wallis, A. 1952. Use of ranks in one-criterion variance analysis. Journal of the American Statistical Association 47: 583-621.

Levin, D. 2002. The role of chromosomal change in plant evolution. Oxford Series in Ecology and Evolution. Oxford University Press, New York. 230 pp.

Muñoz, M., Moreira, A. 2003. Alstroemerias de Chile. Diversidad, distribución y conservación. Taller La Era, Santiago. 140 pp.

Navas-Castillo, J., Cabrero, J., CAmacho, J. 1987. Chiasma redistribution in presence of supernumery chromosome segments in grashoppers on the size of the extra segment. Heredity 58: 409-412.

R CORE TEAm. 2017. R: A language and environment for statistical computing. R Foundation for Statistical Computing, Vienna, Austria. URL http://www.R-project.org/.

ReEves, A. 2001. MicroMeasure: a new computer program for the collection and analysis of cytogenetic data. Genome 44: $239-443$.

SAnso, A. 2002. Chromosome studies in Andean taxa of Alstroemeria (Alstroemeriaceae). Botanical Journal of the Linnean Society 138: 451-459.

Recibido: 09.03.2018

Aceptado: 07.08.2018 